\title{
Jaccoud's arthropathy, an unusual manifestation of idiopathic retroperitoneal fibrosis: rapid improvement of symptoms after tocilizumab treatment
}

\author{
M. Benucci', A. Damiani', F. Li Gobbi', V. Grossi², \\ M. Infantino ${ }^{2}$, A. Arena ${ }^{3}$, M. Manfredi ${ }^{2}$ \\ ${ }^{1}$ Rheumatology Unit, San Giovanni di Dio Hospital, Florence, Italy; ${ }^{2}$ Immunology and Allergology Laboratory, \\ San Giovanni di Dio Hospital, Florence, Italy; ${ }^{3}$ Nuclear Medicine, New Hospital, Prato, Italy
}

\section{SUMMARY}

Jaccoud's arthropathy (JA) is a chronic, non erosive, rheumatoid-like deformity associated with rheumatic fever (RF) and systemic lupus erythematosus and with other diseases such as psoriatic arthritis, connective tissue diseases, hypocomplementemic urticarial vasculitis, infections, sarcoidosis and neoplasia. We described a case of JA in a patient with cutaneous psoriasis but with a particular disease evolution associated with idiopathic retropritoneal fibrosis (IRF), evaluated with computed tomography, magnetic resonance and 18F-FDG PET/ CT. The patient, following failure with steroids, methotrexate and etanercept, was treated with tocilizumab (8 $\mathrm{mg} / \mathrm{kg}$ ) once every 4 weeks for 6 months. A rapid improvement of symptoms and disappearance of 18F-FDG uptake was shown. We describe a review of literature of rheumatic manifestations of IRF and the possible role of interleukin-6 in the pathway of JA and IRF.

Key words: Jaccoud's arthropathy; Idiopathic Retroperitoneal fibrosis; Tocilizumab.

\section{INTRODUCTION}

Taccoud's arthropathy (JA) is a syndrome $\checkmark$ characterised by chronic progressive painless deformity of the hands and feet with surprisingly well-preserved function. JA is a chronic, non erosive, rheumatoidlike deformity associated with rheumatic fever (RF) and systemic lupus erythematous (SLE) and with other diseases such as psoriatic arthritis, connective tissue diseases, hypocomplementemic urticarial vasculitis, infections, sarcoidosis and neoplasia. In general, its prevalence in either SLE or RF is around $5 \%$. The etiopathogenic mechanisms of JA are not known, but some authors have suggested an association with hypermobility syndrome. Several studies have attempted to identify an association between different antibodies and JA in SLE patients, but their findings have not led to any definite conclusion (1). JA is a condi- tion clinically characterised by reversible joint deformities such as swan neck, thumb subluxation, ulnar deviation, boutonniere and hallux valgus, along with absence of articular erosions on the plain radiographs (2). We describe a case of JA in a patient with cutaneous psoriasis but with a particular evolution of the disease associated with Idiopathic Retropritoneal Fibrosis (IRF).

\section{CASE REPORT}

In January 2012 a 37 year-old male patient was admitted to our hospital. The patient had a medical history of pain and bilateral metacarpal-phalangeal joints swelling with ulnar deviation and a deformity boutonniere deformity with hallux valgus in the feet. Because of a history of cutaneous psoriasis he was treated in the past with prednisone $12.5-25 \mathrm{mg}$, methotrexate $15 \mathrm{mg} /$ week for six months and, since these thera- 
pies failed, he started in March 2010 etanercept $50 \mathrm{mg} /$ week for 10 months without results. The patient also complained of fatigue and lumbar pain, which was evaluated as inflammatory lombalgia and he has been treated with NSAID (diclofenac 150 $\mathrm{mg}$ day). His blood examination values were: ESR $45 \mathrm{~mm} / \mathrm{h}, \mathrm{CRP} 1.85 \mathrm{mg} / \mathrm{dl}$ (nv $<0.5 \mathrm{mg} / \mathrm{dl}$ ), Hb $10.5 \mathrm{~g} / \mathrm{dl}$, Ferritin $244 \mathrm{ng} /$ $\mathrm{ml}$. The hands X-Ray showed a deformity of the metacarpal-phalangeal joints with ulnar deviation without erosions (Figure 1). The patient showed no autoantibodies, including ANA, anti ENA, anti ds DNA, ASCA, AGA, EMA, rheumatoid factor and ACPA. The genetic study of HLA showed a double eterozigosis for DQb1-02 DQA105 and DRb1-03. For the persistence of low back pain the patient was submitted to CT scan and MRI, which showed a solid mass in the vertebral body of L5 and in the pre-sacral region with bilateral extension to the foramina obturatoria and compression of nerve roots of L5-S1. Mesenteric lymphoadenitis were also present as well as an involvement of external and internal iliac lymphnodes. Moreover the para-aortic chain was sized 15-20 mm.

The MRI showed also oedema of tissues with enhancement in T2 and extension to the rectum. The patient was submitted to lymphonode biopsy, which showed only angioplastic inflammation with hystiocites, eosinophilis and sclerohyalinosis.

The patient was investigated with (18) FFDG PET/CT, which showed a pre-sacral up-take with extension to L5-S1 and to the left abdomen with ureteral obstruction (Figure 2). The diagnosis of JA (3) in IRF was performed. The serum level of IL-6 was 8.6 $\mathrm{pg} / \mathrm{ml}$ (Human IL6 Instant Enzyme-linked immunosorbent assay (ELISA), Bioscience, Bender MedSystems, GmbH, Vienna, Austria) (nv $<3 \mathrm{pg} / \mathrm{ml})$. The patient began a therapy with tocilizumab $8 \mathrm{mg} / \mathrm{kg}$ every month, maintaining the therapy with prednisone $10 \mathrm{mg}$ and methotrexate $10 \mathrm{mg} /$ week. After two months, prednisone was tapered to $5 \mathrm{mg} /$ day and after four months it was stopped. After six months we repeated 18FDG-PET/CT, which did not show (18)F-FDG uptake (Figure 3). Blood ex-

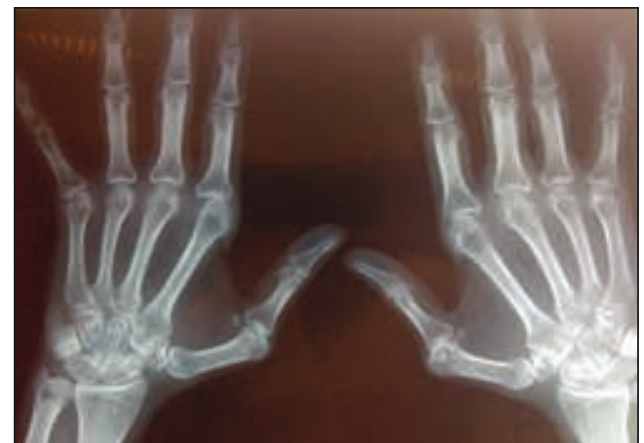

Figure 1 - X-ray showing bilateral metacarpalphalangeal joints swelling with ulnar deviation.
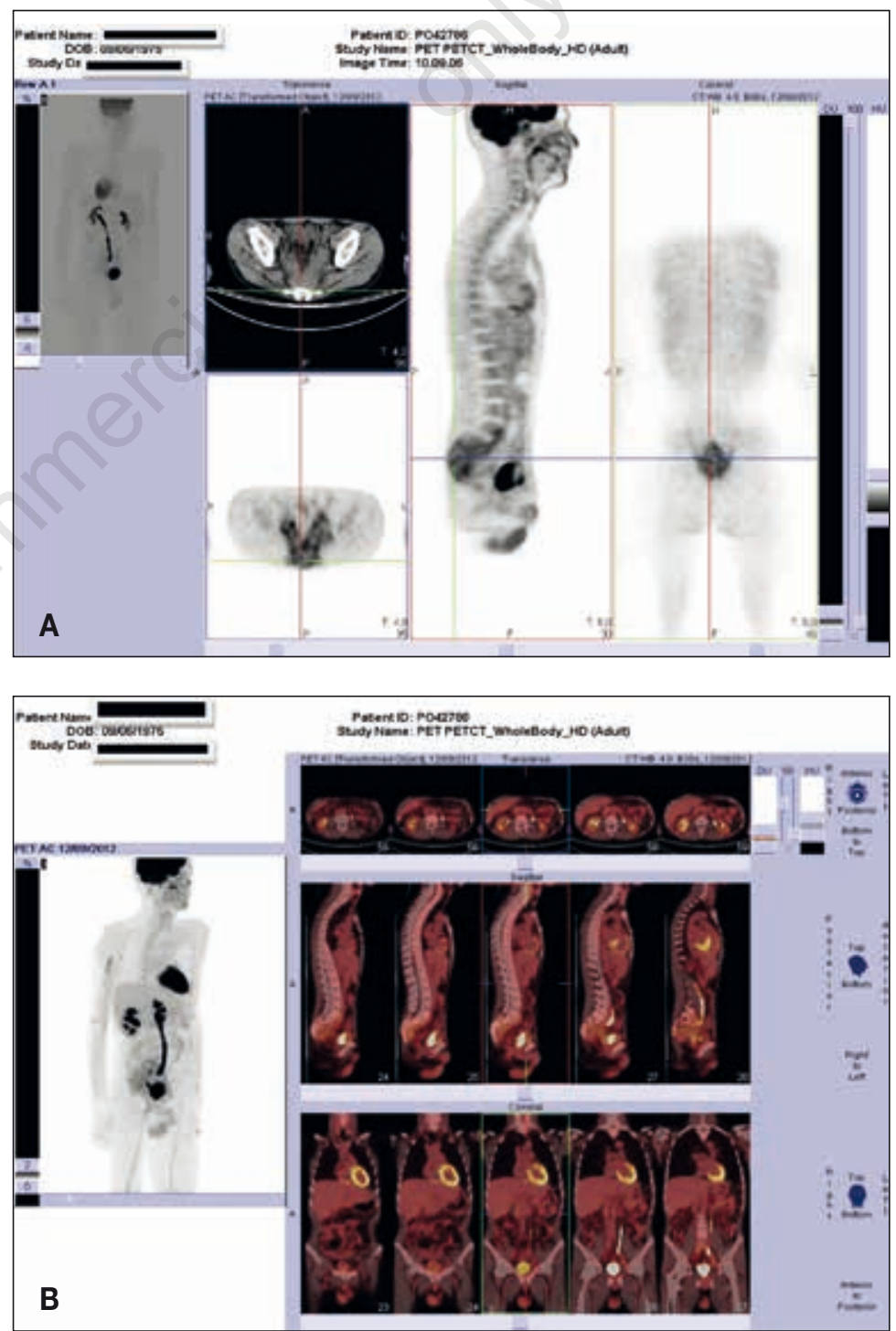

Figure 2 - A) 18F-FDG-PET/CT showing pre-sacral up-take with extension to L5-S1 and B) to the left with ureteral obstruction. 


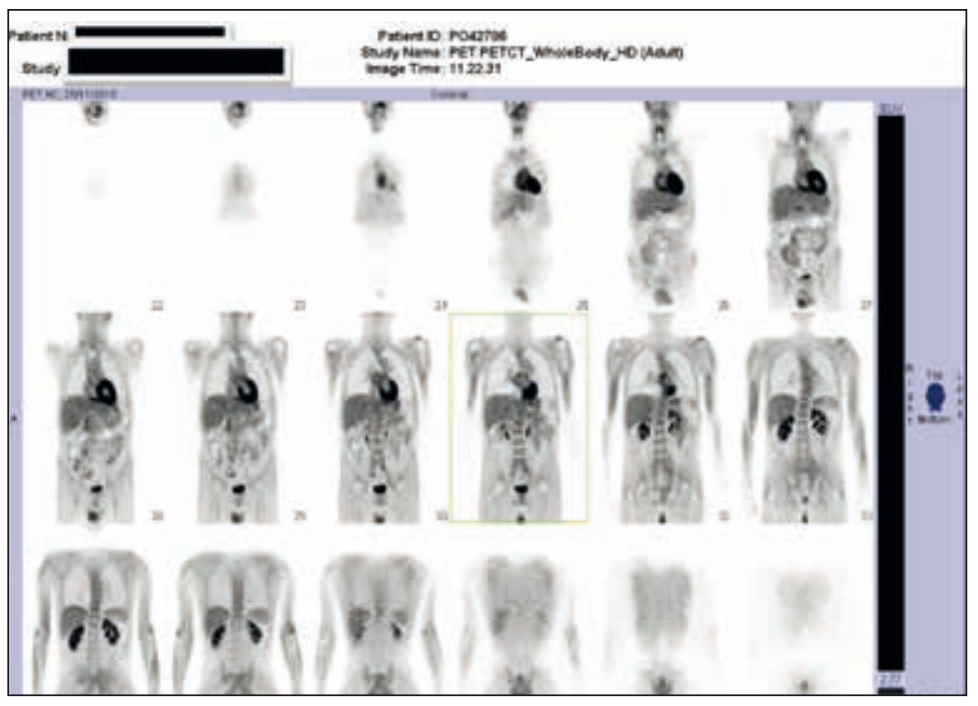

Figure 3 - 18F-FDG-PET/CT enhancing an important reduction of the 18FDG uptake after six months of treatment with Tocilizumab.

ams showed ESR $2 \mathrm{~mm} / \mathrm{h}$, CRP $0.5 \mathrm{mg} / \mathrm{dl}$, IL-6 $<3 \mathrm{pg} / \mathrm{ml}$.

The patient maintains therapy with tocilizumab $8 \mathrm{mg} / \mathrm{kg}$ month and methotrexate 10 $\mathrm{mg} /$ week.

\section{DISCUSSION AND CONCLUSIONS}

The case reported represents the first report in the current literature describing an association between JA and IRF. IRF encompasses a group of rare diseases, whose common denominator is retroperitoneal periaortic fibro-inflammatory tissue, which frequently obstructs neighbouring structures (4). Histology shows a collagen-rich background and fibroblasts admixed with an inflammatory infiltrate consisting of lymphocytes, plasma-cells, macrophages and eosinophils (5). Chronic periaortitis (CP) includes nonaneurysmal [i.e. IRF] and aneurysmal forms [i.e. inflammatory abdominal aortic aneurysm (IAAA), perianeurysmal retroperitoneal fibrosis] (5). A number of findings such as the association with HLA-DRB $1 * 03$, the presence of systemic symptoms and the overlap with other autoimmune diseases, suggest that it may be driven by a systemic autoimmune process (6). In a study of 49 consecutive CP patients who also had peripheral inflammatory arthritis, three devel- oped RA, one had palindromic rheumatism and one suffered from reactive arthritis despite the administration of steroids (7). The review of the English-language literature provided 20 cases, most of them being isolated case reports, showing an association between $\mathrm{CP}$ and arthritis. Fourteen of these patients had spondyloarthritis, five were HLA-B27-positive, whereas other forms such as RA were extremely rare (7). The overlap between IRF and RA suggests that these two diseases may follow common immune-mediated pathways. The presence of autoantibodies, the association with HLADRB $1 * 03$ and the abundance of B cells in the retroperitoneal lesions also indicate that, as in RA, B cells play a major role in CP. Similarities between $\mathrm{CP}$ and RA are also found by examining the composition and architectural organisation of the inflammatory infiltrate in the diseased sites. As in RA, the key players of the inflammatory infiltrate in $\mathrm{CP}$ lesions are B-and T-cells, plasma cells and macrophages (7). Both RA and $\mathrm{CP}$ have a restricted HLA repertoire, although the disease-associated alleles are different (HLA-DRB $1 * 04$ and HLA-DRB1*03, respectively) (8). Studying the genetic background in JA also seems to be of paramount interest. It is known that patients with RF have higher prevalence of HLA-DR7 and DRw53. There are only a few reports on the histopathologic features of JA. Although the presence of synovitis has been described, in general it is mild and without marked synovial membrane proliferation (9). Recently, to explain the non-erosive nature of JA, an experimental model has been proposed. It was based on the information that IFN- $\alpha$ may partecipate in the pathogenesis of SLE arthritis. In this model, the authors proposed an IFN- $\alpha$ biased myelopoiesis: exposure to elevated systemic IFN- $\alpha$ increases the myeloid dendritic cells differentiation to present auto-antigens cells at the expense of osteoclastogenesis in a mutually exclusive manner, as these cells are derived from the same myelomonocyte precursor. Thus, focal erosion becomes impossible due to the lack of mature osteoclasts (9). A recent paper showed that tocilizumab may be a therapeutic option for CP. The systemic and tissue 
up-regulation of IL-6 in CP, together with the clinical benefit of IL-6R blockade observed in 2 patients, suggest that IL-6 may contribute to CP pathogenesis (10).

IL-6 is a pleiotropic molecule, and its functional effects may be relevant for $\mathrm{CP}$ pathogenesis. IL-6 induces activation and differentiation of B cells, which are abundant in the pathologic tissue of $\mathrm{CP}$, where they form the core of the inflammatory aggregates, sometimes organized in germinal centers. IL-6 also promotes differentiation of CD4+ cells into IL17-producing T helper cells, and activates CD8+ cells (10). $\mathrm{T}$ cells, particularly CD4+ T cells, are the most frequent cell type in $\mathrm{CP}$ inflammatory infiltrates, although their $\mathrm{T}$ helper polarization is still unknown. Finally, IL-6 induces fibrosis, a key feature of $\mathrm{CP}$, through different mechanisms, including fibroblast to-myofibroblast differentiation, collagen deposition, and inhibition of metalloproteinase activity (10). Our patient presented a history of skin psoriasis but did not show a flare after the onset of IRF as also shown in a clinical series (11).

This case report, to the best of our knowledge, is the first to suggest an association between JA and IRF.

The overlap between JA and IRF suggests that these two diseases may follow common genetic and immune-mediated pathways. The result obtained after tocilizumab therapy suggests that IL-6 may contribute to the pathogenesis of both diseases.

\section{REFERENCES}

1. Santiago MB. Miscellaneous non-inflammatory musculoskeletal conditions. Jaccoud's arthropathy. Best Pract Res Clin Rheumatol. 2011; 25: 715-25.

2. Santiago MB, Galvão V. Jaccoud arthropathy in systemic lupus erythematosus: analysis of clinical characteristics and review of the literature. Medicine (Baltimore). 2008; 87: 37-44.

3. Santiago MB. Jaccoud's arthropathy: proper classification criteria and treatment are still needed. Rheumatol Int. 2013; 33: 2953-4.

4. Vaglio A, Buzio C. Chronic periaortitis: a spectrum of diseases. Curr Opin Rheumatol. 2005; 17: 34-40.

5. Vaglio A, Salvarani C, Buzio C. Retroperitoneal fibrosis. Lancet 2006; 367: 241-51.

6. Martorana D, Vaglio A, Greco P, et al. Chronic periaortitis and HLA-DRB $1 * 03$ : another clue to an autoimmune origin. Arthritis Rheum. 2006; 55: 126-30.

7. Vaglio A, Palmisano A, Ferretti S, et al. Peripheral inflammatory arthritis in patients with chronic periaortitis: report of five cases and review of the literature. Rheumatology. 2008; 47: 315-8.

8. Goronzy JJ, Weyand CM. Rheumatoid arthritis. Immunol Rev. 2005; 204: 55-73.

9. Mensah KA, Mathian A, Ma L, et al. Mediation of nonerosive arthritis in a mouse model of lupus by interferon-alpha-stimulated monocyte differentiation that is nonpermissive of osteoclastogenesis. Arthritis Rheum. 2010; 62: 1127-37.

10. Vaglio A, Catanoso MG, Spaggiari L, et al Interleukin-6 as an inflammatory mediator and target of therapy in chronic periaortitis. Arthritis Rheum. 2013; 65: 2469-75.

11. Famularo G, Palmisano A, Afeltra A, et al. Retroperitoneal fibrosis associated with psoriasis: a case series. Scand J Rheumatol. 2009; 38: 68-9. 